\title{
Optically Thick Radio Cores of Narrow-Waist Bipolar Nebulae
}

\author{
T.-H. Lee \\ National Optical Astronomy Observatory, Tucson, AZ 85719 \\ and \\ Departemnt of Physics and Astronomy, University of Calgary, Calgary, AB T2N 1N4, \\ Canada \\ thlee@noao.edu \\ J. Lim \\ Institute of Astronomy \& Astrophysics, Academia Sinica, Taipei 106, Taiwan \\ jlim@asiaa.sinica.edu.tw \\ and \\ S. Kwok \\ Department of Physics, Faculty of Science, University of Hong Kong, Hong Kong \\ and \\ Departemnt of Physics and Astronomy, University of Calgary, Calgary, AB T2N 1N4, \\ Canada \\ sunkwok@hku.hk
}

\begin{abstract}
We report our search for optically thick radio cores in sixteen narrow-waist bipolar nebulae. Optically thick cores are a characteristic signature of collimated ionized winds. Eleven northern nebulae were observed with the Very Large Array (VLA) at $1.3 \mathrm{~cm}$ and $0.7 \mathrm{~cm}$, and five southern nebulae were observed with the Australia Telescope Compact Array (ATCA) at $6 \mathrm{~cm}$ and $3.6 \mathrm{~cm}$. Two northern objects, 19W32 and M 1-91, and three southern objects, He 2-25, He 284 and $\mathrm{Mz} 3$, were found to exhibit a compact radio core with a rising spectrum consistent with an ionized jet. Such jets have been seen in M 2-9 and may be responsible for shaping bipolar structure in planetary nebulae.
\end{abstract}

Subject headings: planetary nebulae: general — stars: AGB and post-AGB stars: winds, outflows — stars: mass loss — stars: evolution — radio continuum: stars 


\section{Introduction}

Planetary nebulae (PNs) show a remarkably broad range of often intricate morphologies. Butterfly nebulae, classified as bipolar nebulae with a narrow pinched waist at the center (Balick \& Frank 2002), are probably the most spectacular, and yet at the same time most puzzling. Under the classical interacting stellar winds (ISW) model (Kwok et al. 1978), a PN is formed when the fast ionized wind from its central star overtakes the material ejected earlier by the slow neutral wind and compresses the material into a dense shell. This produces a round PN if both winds are isotropic. A dense equatorial torus, which blocks the fast wind in that direction, is added to the interacting winds system to explain how asymmetric structure in PNs (e.g., bipolar) can be developed (Kahn \& West 1985; Balick 1987). This, however, can only produce bipolar nebulae with wide waists, not those with narrow "pinched" waists. It has been suggested that the creation of the latter nebulae requires a collimated fast wind (e.g., Soker \& Rappaport 2000). Soker \& Rappaport's model invokes a binary star system to produce a collimated fast wind. The system consists of a mass-losing AGB star and a main-sequence or white dwarf companion. The AGB wind captured by the companion forms an accretion disk around the companion that loads and drives a collimated fast ionized wind.

The existence of collimated outflows (COFs) has been suggested by numerous observations, usually from the ionized gas images. For example, the highly collimated lobes of some PNs and PPNs (proto-planetary nebulae) imply that their morphology is shaped by a collimated outflow (see review by Kwok 2003a, and references therein). The first discovery of strongly collimated high-velocity bipolar outflows in a PN was by Gieseking et al. (1985), in NGC 2392, the Eskimo nebula. They analyzed from spectroscopic observations of this nebula, particularly in the [N II] $\lambda 6583$ emission line, they found a set of bipolar jets with a expansion velocity of $\sim 200 \mathrm{~km} \mathrm{~s}^{-1}$ and a small angle of collimation less than $10^{\circ}$. Although unexpected at first, the pervasiveness of COFs in PNs is now widely recognized. COFs have diverse characteristics. They are prominent in emission lines such as $[\mathrm{N} \mathrm{II}]$ and $[\mathrm{S}$ II] and are observed as bipolar, multipolar, point-symmetric and jet-like outflows. The expansion

velocities of these COFs range from several tens to a thousand $\mathrm{km} \mathrm{s}^{-1}$ (see reviews of López 2002, 2003).

In addition to ionized gas, COFs have also been seen in neutral gas. Several authors have made detailed studies on CO emission lines of individual PNs and PPNs (e.g., Forveille et al. 1998; Huggins et al. 2000). The velocities for these CO outflows are found to be much smaller than those of the ionized COFs. Bujarrabal et al. (2001) studied the CO emission from a large sample of PPNs as well as two yellow hypergiants and one young PN. They reported collimated fast outflows in 28 of 32 objects, indicating that such outflows are a common phenomenon in the early PN stage. 
In the model proposed by Soker \& Rappaport (2000), the collimated winds responsible for shaping butterfly nebulae are formed and collimated close to the central star system. They are difficult to observe in optical wavelengths directly, because dust around the equator limits the depth into the center that optical observations can probe. The dust is optically thin to radio emission, which can therefore probe deep into the center to look for central jets, if the jets are at least partially ionized. Using the VLA, Lim \& Kwok (2000, 2003) have indeed found a collimated wind in the form of a pair of ionized jet in the core region of the narrow-waist bipolar nebula M 2-9.

M 2-9 has a bright central core and two cigar-shaped lobes extending from opposite sides of the core $\sim 20^{\prime \prime}$ to the north and south. The lobes of the nebula contain three pairs of symmetric knots or bright features: two of them are with mirror symmetry and one pair with point/mirror symmetry. Because of its spectacular morphology, M 2-9 is one of the most well-studied planetary nebulae. Since its discovery in 1947, the core and the outline of the lobes have remained stationary, but the knots and bright features have moved laterally from the west to the east (e.g., Allen \& Swings 1972; Kohoutek \& Surdej 1980). Various models have been proposed to explain the lateral motions of bright features of M 2-9. For example, Allen \& Swings (1972) proposed that the changes were the result of a rotating beam of ionizing radiation from the star shining through a matched pair of holes in orbiting clouds. Kohoutek \& Surdej (1980) noted that it is unlikely such model can result in positional changes only in the east-west direction, and instead suggested that the nebula was rotating as a whole. However, since Goodrich (1991) concluded that the distance of M 2-9 cannot be only 50 pc as proposed by Kohoutek \& Surdej (1980), the unreasonable large velocities of the knots have made their model problematic.

Recently in explaining the structural changes of M 2-9, Doyle et al. (2000) and Livio \& Soker (2001) both invoke a symbiotic star system to produce a mirror-symmetric jet. Direct evidence of such a jet is provided by observations of M 2-9 at $6 \mathrm{~cm}, 3.6 \mathrm{~cm}, 2 \mathrm{~cm}$, and $1.3 \mathrm{~cm}$ by (Lim \& Kwok 2003). Their observations were largely insensitive to the large-scale free-free emission from the nebula and focused instead on the compact radio core at the center found by Kwok et al. (1985). Their results indicate that this optically thick core has a spectral index of $0.67 \pm 0.01$, which is close to the spectral index of 0.6 expected from an isothermal outflow expanding at a constant velocity and opening angle (Reynolds 1986). More importantly, at the shortest wavelength of $1.3 \mathrm{~cm}$ where the jet is best resolved, its bipolar lobes show a mirror-symmetric structure (Figure 1 of Lim \& Kwok 2003). Both lobes point in the directions of mirror-symmetric brightenings in the nebular walls seen in the HST image observed just 2 years earlier (N1 and S1 in Figure 2 of Doyle et al. 2000). The qualitative properties of this jet are in good agreement with those predicted by Livio \& Soker (2001). 
Motivated by the discovery of the ionized jets in $\mathrm{M} 2-9$, we launched a search for collimated ionized winds in butterfly nebulae using the VLA and the ATCA. We selected a total of 16 objects from Table 1 of Soker \& Rappaport (2000) to search for optically thick radio cores - a characteristic signature of ionized jets. The selected objects were classified by Soker \& Rappaport (2000) to have either narrow waists (10 objects) or possibly narrow waists (6 objects), whose morphology cannot be determined with absolute certainty based on the optical images obtained by Corradi \& Schwarz (1995). We are interested in finding any central source that is optically thick at short wavelengths where the nebular material is already optically thin. Our goal is to identify suitable candidates for follow-up observations with higher sensitivity and resolution to reveal the structure of the source. We observe each nebula with two wavelengths to calculate its spectral index, which tells us if the emission is optically thick. In this paper, we report the results of our search for the radio cores. The observations and data reduction are described in Section 2, In Section 3, we report that radio emission were detected in 9 sources, and that 5 of those detected have optically thick radio cores. Section 4 discusses the implication of the high mass loss rates derived from the flux densities of the radio cores. Lastly, a summary is given in Section 5 .

\section{Observations and Data Reduction}

The survey sample comprises 11 northern objects (Declination $\geq-40^{\circ}$ for the VLA observation limits), IRAS 07131-0147, M 1-16, NGC 2818, NGC 6302, 19W32, HB 5, NGC 6537, M 3-28, M 1-91, M 2-48, and NGC 7026, observed with the VLA at $1.3 \mathrm{~cm}$ and $0.7 \mathrm{~cm}$, and 5 southern objects, He 2-25, He 2-36, He 2-84, Th 2-B, and Mz 3, observed with the ATCA at $6 \mathrm{~cm}$ and $3.6 \mathrm{~cm}$. The observing wavelengths are the shortest wavelengths feasible at the time of observations, which give the highest angular resolutions possible. The optical properties of the objects are listed in Table 1, with columns 1 and 2 giving the common name and the PN G name based on galactic coordinates, columns 3 and 4 giving Right Ascension and Declination, column 5 indicating if they appear to have a very narrow waist, column 6 stating if the PN has a compact central source visible in optical images, and columns 7 indicating if the nebula shows point or mirror symmetric structure. These properties are mainly from Soker \& Rappaport (2000), with some update obtained from recent optical images. A question mark means the attribute cannot be determined from available images. 


\subsection{VLA Observations}

The 11 northern objects were observed at $1.3 \mathrm{~cm}$ and $0.7 \mathrm{~cm}$ with the A-configuration in 2002 March 10. The A-configuration is the most extended configuration, hence it provide the highest angular resolution achieved with the VLA. It allows us to not just look for but resolve if possible a central compact and optically think source, as well as to better discriminate against if not resolve out extended nebular emission. The observations were conducted in fast-switching mode with a calibration cycle time of just 2-3 minutes in order to correct for rapid phase variation at such short wavelengths. The integration time for the individual sources is about 20 minutes each at $1.3 \mathrm{~cm}$ and $0.7 \mathrm{~cm}$. 3C286 was observed for about 5 minutes in order to calibrate the flux scales for individual sources. The data were reduced and analyzed using the package AIPS following the standard procedures for reducing highfrequency data outlined in Appendix D of the AIPS Cookbook (NRAO 2003). The calibrated data were then Fourier transformed, weighted and CLEANed to generate the final CLEAN images. Natural weighting was applied in order to obtain images with minimum-noise levels. We achieved an angular resolution of $\sim 0.08^{\prime \prime}$ at $1.3 \mathrm{~cm}$ and $\sim 0.04^{\prime \prime}$ at $0.7 \mathrm{~cm}$ for the final CLEAN maps.

\subsection{ATCA Observations}

The 5 southern objects were observed at $6 \mathrm{~cm}$ and $3.6 \mathrm{~cm}$ with the $6-\mathrm{km}$ " $6 \mathrm{C}$ " configuration in 2003 March 15 and 16. These two wavelengths were the shortest wavelengths feasible when the observations took place. With $6-\mathrm{km}$ being the most extended configuration, they provide the highest angular resolution achievable at the time of observation. We observed each source for 15 minutes roughly every 40 minutes over a period of 12 hours in order to optimize the $u v$-coverage, obtaining a total integration time of 4-5 hours on each object. The primary calibrator 1934-638 was observed for flux calibration. The data were reduced using the reduction package MIRIAD following the procedures outlined in Miriad Users Guide (Sault \& Killeen 2003). The final CLEAN images were obtained by performing inverse Fourier transforms from the calibrated visibility datasets to images, and then deconvolve the sidelobes using the CLEAN algorithm. Natural weighting was used and we attained an angular resolution of $\sim 3^{\prime \prime}$ at $6 \mathrm{~cm}$ and $\sim 1.5^{\prime \prime}$ at $3.6 \mathrm{~cm}$. Where applicable, the self-calibration was performed to improve the image quality. In the end, only He 2-84 has strong enough emission to allow phase self-calibration. 


\subsection{Optical Observations}

Three objects, He 2-36, He 2-84, and 19W32, which have radio emission detected in our study but have no high resolution optical images, were observed with the $2.3 \mathrm{~m}$ Telescope in Siding Spring Observatory (SSO) in 2003 May 26-30. The Imager instrument and three narrow-band filters, $\mathrm{H} \alpha,[\mathrm{N}$ II] and [O III] were used to take photometric images. The center wavelength and bandwidth of each filter are listed in Table 2, along with the best achieved FWHM during the observations. These filters' bandwidths of $\leq 10 \AA$ allow minimize contaminations from adjacent emission lines. The CCD has dimensions of $1024 \times 1024$, with a focal plane scale of $0.59^{\prime \prime} /$ pixel determined by the telescope optics.

The images were reduced with standard techniques using IRAF software (Massey 1997). The averaged bias frames and the overscan level were subtracted from each target image, which was then trimmed to discard the overscan region and the first and last few columns and rows. The dark current was negligible during the observations, so no dark current subtraction was performed. Finally, the images were divided by the flat-field image for each filter. The exposure frames of each object were then combined to generate the final images in each filter, using a statistical method that removes cosmic ray contamination.

\section{Results and Analyses}

Among the 16 objects observed, we detected radio emission in nine of them, and five of which have a central compact source. In Table 3 we list the detect limits $(3 \sigma)$ of our observations, and the type of emission that were detected. A more detailed description of the detections and the analysis for the compact sources are given in the following subsections.

\subsection{Northern Survey}

Of the 11 objects observed by VLA, we found that NGC 6302, Hubble 5 and NGC 6537 only exhibit extended radio emission at $1.3 \mathrm{~cm}$ that could not be properly mapped due to the lack of short baselines in our observations. Two objects, $19 \mathrm{~W} 32$ and M 1-91, show only a compact central source at both $1.3 \mathrm{~cm}$ and $0.7 \mathrm{~cm}$. No emission was detected for the remaining six objects. This is the first time the centers of all these nebulae have been examined at such high angular resolutions at radio wavelengths. Note that for the six objects with non-detection in our observations, some have been detected at $6 \mathrm{~cm}$ by Zijlstra et al. (1989) and Aaquist \& Kwok (1990), whose surveys are more sensitive to the extended emission. 
Here we present the images for the radio cores of 19W32 and M 1-91, along with their optical images (Figures 1 and 2). Both objects exhibit a compact stellar-like optical core. We obtained the optical images of $19 \mathrm{~W} 32$ from SSO $2.3 \mathrm{~m}$ telescope observations as described above, those of M 1-91 from the IAC (Instituto de Astrofísica de Canarias) catalog (Manchado et al. 1996). All images are oriented with north up and east to the left.

\subsubsection{W32}

The H $\alpha$ and [N II] images of 19W32 show a prominent stellar-like optical core and two lobes in opposite directions, extending $\sim 10^{\prime \prime}$ to the northeast and southwest. Only the $\mathrm{H} \alpha$ image is presented in Figure 1, No [O III] emission was detected for this nebula. The radio continuum images only show a compact core, which coincides with the optical stellar core.

\subsection{2. $M$ 1-91}

The $\mathrm{H} \alpha,[\mathrm{N}$ II] and [O III] images of M 1-91 shows an optical core and two lobes in opposite directions, extending at least $20^{\prime \prime}$ to the northeast and southwest. Since the images of the three filters show similar structures, only the $\mathrm{H} \alpha$ image is presented in Figure 2. M 191's morphology is very similar to M 2-9, except that the brightenings of the lobes being point symmetric instead of mirror symmetric. The radio continuum images only show a compact core, which coincides with the optical stellar core. The $1.3 \mathrm{~cm}$ emission of M 1-91 shows some extended structures that might align with the point-symmetric structures. This needs further observations to confirm.

\subsubsection{Spectral Indices of the Radio Cores}

The spectral index, defined as the slope of the radio spectrum $\frac{d \log F_{\nu}}{d \log \nu}$, indicates the emission mechanisms and optical depths of the radio emission. Optically thick free-free emission has a rising spectrum, optically thin free-free emission has a flat spectrum $(\sim-0.1)$, and optically thin nonthermal emission has a steeply falling spectrum. In the case of a spherically symmetric nebula, the radio emission has a spectral index of $0.6\left(S_{\nu} \propto \nu^{0.6}\right)$ for an isothermal stellar wind with a constant expansion velocity (Wright \& Barlow 1975; Panagia \& Felli 1975). For a special case of collimated ionized winds with constant opening angle, the radio emission also has a spectral index of 0.6 (Reynolds 1986).

In order to determine the properties of the radio cores of $19 \mathrm{~W} 32$ and M 1-91, we cal- 
culated the spectral indices of their radio emission. To determine the flux densities of the radio cores observed in 19W32 and M 1-91, a two-dimensional Gaussian structure was fit to the measurements by applying the AIPS tasks IMFIT to the clean map and OMFIT to the visibility data. Because of the low $\mathrm{S} / \mathrm{N}$ ratio in these observations, neither task gives an obviously superior fit. Therefore results from both tasks are given. The entire dataset were used for fitting at both 1.3 and $0.7 \mathrm{~cm}$. We also fit a more restricted dataset at $0.7 \mathrm{~cm}$ chosen to have the same sampling range in angular scales as at $1.3 \mathrm{~cm}$ to check for a greater inclusion of any extended nebula emission in the larger synthesized beam. We found that both procedures gave the same results within measurement uncertainties $(<1 \sigma)$. Thus, in Table 4, only the results obtained by fitting the datasets over their entire $u v$ range are listed. The rising spectra of both 19W32 and M 1-91 suggest that their radio cores are produced by optically thick free-free emission. Fits to the measured visibilities show that these cores are resolved along one dimension; i.e., the minor axis of the synthesized beam. The size of the emitting source will be discussed further in $\$ 4.1$

\subsection{Southern Survey}

Of the five objects observed by ATCA, He 2-36 shows only extended emission, He 2-25 and He 2-84 shows only a compact central source, Mz 3 has both, and no emission was detected from Th 2-B. Figures 3 to 6 present the radio and optical images of the four objects with detections. For the three objects with radio core detections, their optical images all show a compact stellar-like cores. The optical images of He 2-25 were obtained from Lee et al. (2006), those of Mz 3 from the HST archive, and those of He 2-36 and He 2-84 from SSO $2.3 \mathrm{~m}$ telescope observations. As before, images are oriented with north up, east to the left.

\subsubsection{He 2-25}

The optical images of He 2-25 display a bright central source and a pair of very faint bipolar lobes extending toward north and south (Figure 3). The radio emission of this object is only detected at $3.6 \mathrm{~cm}$, which shows a central unresolved source coinciding with the optical core. 


\subsubsection{He 2-36}

The optical images of He 2-36 display a bright central source and an equatorial torus with limb brightening arms extending clockwise from the torus edges toward the north and south. The brightening resembles an S-shape point symmetrical distribution. We presented the $\mathrm{H} \alpha$ image in two different scales in order to show the detailed structures (Figure 44). The nebula possesses a more moderate degree of bipolarity (lobes are less collimated) than other butterfly nebulae observed in this work. The radio images only show emission from the equatorial band. Although the radio images show some interesting features, these are not relevant to current investigation.

\subsection{3. $\mathrm{He} 2-84$}

The $\mathrm{H} \alpha$ and [N II] images of He 2-84 show an optical core with an equatorial torus. The two lobes extending at right angles to this torus toward the northeast and southwest appear to have a point symmetrical geometry. The $\mathrm{H} \alpha$ image is displayed in two different scales in order to show the detailed structures (Figure 5). The lobes are very faint and cannot be seen in the [O III] image. The radio continuum images only show a central unresolved bright source, which coincides with the optical core.

\subsection{4. $M z 3$}

The optical images of $\mathrm{Mz} 3$ show a bright core, two approximately spherical bipolar lobes, and even more extensive filamentary nebulosities on either side of the central star can be seen from its $\mathrm{H} \alpha$ and [N II] images. Only the $\mathrm{H} \alpha$ images is presented in Figure 6. The total size of the nebula spans over $50^{\prime \prime}$ on the sky. The radio images of $\mathrm{Mz} 3$ show emission

from the central source and the two lobes, similar to the radio images of M 2-9 (Kwok et al. 1985). The radio central source and the two lobes coincide with the optical core and the brightest structures of the optical lobes.

\subsubsection{Spectral Indices of the Radio Cores}

He 2-25 has a compact radio core detected only at $3.6 \mathrm{~cm}$. The fact that no detection at $6 \mathrm{~cm}$ has already indicated that this core is optically thick. Using the detection limit at $6 \mathrm{~cm}$, a lower limit of spectral index is estimated to be 1.25. However, a more precise evaluation 
of the spectral index of He 2-25 has to wait for observations at shorter wavelengths.

He 2-84 and Mz 3 have radio cores detected both at $3.6 \mathrm{~cm}$ and $6 \mathrm{~cm}$, suitable for spectral index determination. For He 2-84, we applied the task IMFIT to the clean map and UVFIT to the visibility data to determine the flux densities of its radio core assuming the radio core to have a two-dimensional Gaussian structure. For Mz 3, the flux density was obtained only for the bright compact core in the CLEAN map. This core can be seen in Figure 6. The total flux density was obtained by summing up the flux densities of the CLEAN components in the radio core region, since the convolution of the Clean components with the CLEAN beam might result in the confusion of the core emission and nebular emission. The results are listed in Table 5 with $1 \sigma$ uncertainties. Even with only clean components in the core region considered, it seems only part of the diffuse emission is excluded. An analysis of this is given later.

The radio core of He 2-84 has a spectral index significantly more negative than the value of -0.1 expected for optically thin free-free emission. This suggests that the emission of the observed radio core is dominated by more extended emission that is, as expected, better resolved out at shorter than at longer wavelengths. Therefore the extended emission makes a stronger contribution at $6 \mathrm{~cm}$ than at $3.6 \mathrm{~cm}$, resulting in a spectral index that is even more negative than expected for optically thin free-free emission. To illustrate the effects of the extended emission on the flux measurements, Figure 7 plots amplitude vs. uv-distance for He 2-84 at $6 \mathrm{~cm}$ and $3.6 \mathrm{~cm}$. The real component of the amplitudes have been averaged in several $u v$ bins. The binning size is wider at longer $u v$-distance in order to increase the signal to noise ratio at longer baseline. As shown in the figure, the amplitude decreases with increasing $u v$-distance which clear demonstrates that an extended component is present.

In order to mitigate against contamination from the diffuse nebular emission, we excluded the short baselines $(<50 \mathrm{k} \lambda)$ that pick up the extended nebular component. We also excluded the longest baselines at $3.6 \mathrm{~cm}$ in order to provide the same angular resolution as at $6 \mathrm{~cm}$. Because of the noise increases caused by removing $u v$ data, the visibility and map fits did not yield reliable results. Consequently, flux densities were derived by summing the flux densities of the CLEAN components. The results are listed in Table 6, which indeed shows that the extended emission constitutes $\sim 90 \%$ of the flux at $6 \mathrm{~cm}$, and $\sim 80 \%$ of the flux at $3.6 \mathrm{~cm}$ for $\mathrm{He} 2-84$. A similar analysis was also performed on $\mathrm{Mz} 3$, since some contamination from the extended emission is also possible. The results listed in Table 6 show that for $\mathrm{Mz} 3$, the flux contributed from the extended emission is relatively small at $6 \mathrm{~cm}$, and there is virtually no contamination at $3.6 \mathrm{~cm}$.

The differences between the core flux densities and resultant spectral indices obtained using the different $u v$ distances suggest that for both He 2-84 and $\mathrm{Mz} 3$, the emission comes 
from two sources, one compact and one diffuse. In both cases, the more careful analysis incorporating the same $u v$-coverage (Table 6) indicates that an optically thick radio core has been detected. A separate ATCA investigation of the radio properties of Mz 3 by Bains et al. (2004) also concluded that Mz 3 has an optically thick core.

It is worthwhile mentioning that we did find a compact radio source both in $6 \mathrm{~cm}$ and $3.6 \mathrm{~cm}$ inside the primary beam of the Th 2-B observation as shown in Figure 8 . However, after carefully examining the position of this source with the known objects in the field using SIMBAD 1 , we determined that this is a background radio source not related to Th 2-B, since it is about $3^{\prime}$ away from the optical postion of Th 2-B. This background radio source has a spectral index of -1 , characteristic of nonthermal radio emission. It is probably a quasar.

\section{Discussion}

\subsection{The Mass Loss Rate}

The VLA and ATCA radio flux densities obtained in the previous sections can be used to estimate the mass loss rates of the nebulae, following the procedure in Wright \& Barlow (1975). Wright \& Barlow gave the mass loss rate for a spherical wind with a spectral index of 0.6 as

$$
\dot{M}=0.095 \frac{\mu_{e} v S_{\nu}^{3 / 4} D^{3 / 2}}{Z x_{e}^{1 / 2} g^{1 / 2} \nu^{1 / 2}} \quad \mathrm{M}_{\odot} \mathrm{yr}^{-1},
$$

where $v$ is the velocity of the mass loss flow in $\mathrm{km} \mathrm{s}^{-1}, S_{\nu}$ is the flux observed at frequency $\nu$ in Jy, $\nu$ is in $\mathrm{Hz}, D$ is the distance in kpc, $Z$ is the nuclear charge, $\mu_{e}$ is the mean atomic weight per electron, $x_{e}$ is the ratio of electron density to the number density of ionized $\mathrm{H}$, i.e., $n_{e}=x_{e} n_{p}$, and $g$ is the Gaunt factor, which can be approximated in the radio regime as

$$
g(\nu, T)=\frac{\sqrt{3}}{\pi}\left\{17.7+\ln \left[\frac{\left(T_{e} / \mathrm{K}\right)^{3 / 2}}{(\nu / \mathrm{Hz}) Z}\right]\right\},
$$

where $T_{e}$ is the electron temperature (Osterbrock 1989, p. 95).

The constants $x_{e}$ and $\mu_{e}$ can be found by assuming the He to $\mathrm{H}$ number ratio $y$ and the fraction of He that is singly ionized $y^{\prime}$, since the contributions from heavy elements are negligible due to their low abundance. The singly ionized He to $\mathrm{H}$ ratio is $y^{\prime} y$ and the doubly ionized He to $\mathrm{H}$ ratio is $\left(1-y^{\prime}\right) y$ if there is no neutral He. The ratio of electron density to

\footnotetext{
${ }^{1}$ http://simbad.u-strasbg.fr/
} 
the number density of ionized $\mathrm{H}$ becomes $x_{e}=1+y y^{\prime}+2 y\left(1-y^{\prime}\right)$, and the mean atomic weight per electron is

$$
\mu_{e}=\frac{1+4 y}{1+y y^{\prime}+2 y\left(1-y^{\prime}\right)}
$$

For $y=0.11$ and $y^{\prime}=0.5, x_{e}$ and $\mu_{e}$ have values of 1.165 and 1.236 , respectively.

Assuming $T_{e}=4000 \mathrm{~K}$ (the temperature of free electrons are mainly contributed by ionization of hydrogen, Reid \& Menten 1997) and $Z=1$, we calculate the mass loss rate as a function of distance and wind velocity using the flux densities found in our observations. Due to the large uncertainties of the mass loss rate parameters, the differences from the flux densities at different wavelengths for the same object are nebligible. Therefore we give a best estimate of the mass loss rate for each object in Table 7.

To calculate the actual mass loss rates, the wind velocities and the distances to the nebulae are needed. Assuming a typical wind velocity of $1000 \mathrm{~km} \mathrm{~s}^{-1}$, and using the distances available from the literature as given in Table 7, we obtain $\dot{M} \sim 7 \times 10^{-6} \mathrm{M}_{\odot} \mathrm{yr}^{-1}$ for He 2-25, $\dot{M} \sim 10^{-5} \mathrm{M}_{\odot} \mathrm{yr}^{-1}$ for He $2-84, \dot{M} \sim 3 \times 10^{-5} \mathrm{M}_{\odot} \mathrm{yr}^{-1}$ for $19 \mathrm{~W} 32, \dot{M} \sim 9 \times 10^{-5} \mathrm{M}_{\odot} \mathrm{yr}^{-1}$ for M 1-91 and $\dot{M} \sim 2 \times 10^{-4} \mathrm{M}_{\odot} \mathrm{yr}^{-1}$ for Mz 3, respectively. The mass loss rate for $\mathrm{Mz} 3$ is consistent with that derived by Bains et al. (2004). They found $\dot{M} \sim 5-30 \times 10^{-5} \mathrm{M}_{\odot} \mathrm{yr}^{-1}$ using the range of $D$ available in the literature.

We also used the Eq. (11) in Wright \& Barlow (1975) to calculate the size of the emitting source, under the spherical wind assumption. The size equation, when incorporated Eq. (1), reduced to

$$
\begin{aligned}
r(\nu) & =5.83 \times 10^{27} S_{\nu}^{1 / 2} T^{-1 / 2} \nu^{-1} D \quad \mathrm{~cm} \\
& =3.9 \times 10^{11} S_{\nu}^{1 / 2} T^{-1 / 2} \nu^{-1} \quad \operatorname{arcsec}
\end{aligned}
$$

where $r$ corresponds to the radius of the emitting region where the optical depth $\tau_{\nu}=0.244$. For the unresolved sources, Eq. (4) predicts a source size smaller than the synthesized beam. For the two resolved sources 19W32 and M 1-91, we listed the measured dimensions along the resolved axis and the predicted source sizes in Table 8 . The predicted sizes are about 4 or 5 times smaller than the measured dimensions. It is possible that we underestimated the predicted size by giving the wrong assumption of temperature in Eq. (41). However, to make the predicted size consistent with the measured dimension, the temperature has to be unrealistically small $(\sim 200 \mathrm{~K})$, in which case the winds are not ionized. Therefore, we concluded that the predicted sizes are smaller is because these sources are elongated, possibly by collimated winds (jets).

If we approximate a collimated source as an ellipse with major and minor axes of $a$ and 
$b$, and the collimated wind has an emission area as the same as a spherical wind, then

$$
a=r \sqrt{2 / \theta_{0}} \text {. }
$$

where $\theta_{0}=2 b / a$ is the opening angle of the jet in radians. Using the measured dimension of the resolved axis as $a$, we can derive the opening angle of the collimated jet for $19 \mathrm{~W} 32$ and M 1-91 as listed in Table 8. The opening angle can then be used to revise the mass loss rate. As pointed out by Reynolds (1986), a collimated wind with a much lower mass loss rate can easily produce the same radio intensity as an uncollimated wind with a much higher mass loss rate, with the relationship for a spectral index of 0.6 is

$$
\frac{\dot{M}(\text { jet })}{\dot{M}(\text { spherical wind })}=0.20 \theta_{0}(\sin i)^{-1 / 4}
$$

where $i$ is the inclination of the major axis of the wind to our line of sight. Assuming $i=90^{\circ}$, which gives a lower limit of the ratio, we derived the ratio is $\sim 0.02$ in both cases. Thus we revised the mass loss rates of $19 \mathrm{~W} 32$ and M 1-91 to $\dot{M} \sim 10^{-6} \mathrm{M}_{\odot} \mathrm{yr}^{-1}$ for collimated winds, which are about 50 times lower than assuming spherical symmetry. From the analysis of central-star wind P-Cygni profiles observed with the International Ultraviolet Explorer, the

mass loss rates from central stars of PNs are $10^{-9}-10^{-7} \mathrm{M}_{\odot} \mathrm{yr}^{-1}$ (Perinotto 1983). These values are consistent with theoretical values expected from winds driven by radiation pressure on ionized gas (Pauldrach et al. 2003). The mass loss rates we inferred are at least an order of magnitude higher than expected. Such high mass loss rates can be explained if the central stars of these nebulae are symbiotic stars, as in the case suspected for M 2-9 whose inferred mass loss rate is $\sim 6 \times 10^{-6} \mathrm{M}_{\odot} \mathrm{yr}^{-1}$ (Lim \& Kwok 2003). Similarly, Bains et al. (2004) also favored a binary companion scenario instead of the superwind of a single progenitor to explain the observational properties of $\mathrm{Mz} 3$.

\subsection{PNs or Symbiotic Stars?}

Symbiotic stars are interacting binary systems, typically consisting of a white dwarf that accretes material from the wind of a cool red giant companion, sometimes forming a disk around the white dwarf. The radiation from the hot star partially ionizes the wind from the cool star. A spectrum of the two together thus shows a combination of absorption lines from the surface of the cool red giant and emission lines from the nebula. First noticed by Merrill (1919), Plaskett (1928), and Merrill \& Hummason (1932) as stars with combination of spectra, these objects were named symbiotic stars by Merrill (1958).

The possibility of some well-known PNs can in fact be symbiotic stars was discussed by Kwok (2003b) and Corradi (2003). With the discovery of increasing number of PNs with 
binary nuclei, the simplistic notion that PNs have single central stars and symbiotics are binaries is no longer viable. It is often difficult to distinguish the two classes of objects by morphology, spectrum, or spectral energy distribution. Only in cases where periodic photometric variability as a result of pulsation, or the observations of photospheric molecular absorption band in the spectrum, that one can be certain that we are dealing with a symbiotic system. However, it is difficult to rule out that certain PNs, including those under study here, are NOT symbiotic stars. Even the evolutionary status of extremely well-studied objects such as M 2-9 is still not certain.

If these nebulae with radio cores are indeed symbiotic star systems, the ionized wind we measured would originate from the white dwarf companion. The mass loss rate of this wind does not directly tell the mass loss rate of the AGB star, but they must be related since the accretion disk is material captured from the AGB star wind. If there is a balance between accretion onto the disk and ejection to form a jet, then the mass loss rate of the AGB star must be at least as high as the mass loss rate of the jet. Assuming a spherically symmetric outflow and only a fraction of the slow neutral wind from the AGB star is captured by the WD dwarf, the corresponding mass loss rate of the AGB star would be at least in excess of $10^{-4} \mathrm{M}_{\odot} \mathrm{yr}^{-1}$. Such values are seldom observed in AGB stars. This problem can be overcome if the AGB star wind is strongly concentrated in the binary orbital plane, as is predicted for symbiotic star systems with relatively small orbital separations.

The remaining objects have no detected radio core emission because they are either too distant or too faint. None of those with known distances $(0.5-7 \mathrm{kpc})$ are farther than the most distant PN with a detected radio core (M 1-91, $7 \mathrm{kpc}$ ). If all these objects have the same radio luminosity, we should be able to detect all of them. Therefore the more likely explanation is that these objects have intrinsically faint radio emission. It is possible that those objects evolved from single stars thus do not produce strong radio emission. Since the mass loss rate from the central stars of PNs are at least an order of magnitude smaller than that of the symbiotic systems, even if these nebulae do have ionized jets from the central stars, their radio emission would be too faint to be detected. Thus radio observations may provide a way to separate symbiotic nebulae from genuine planetary nebulae. This would also mean that there is more than one mechanism to give a nebula a narrow-waist shape. For single stars, one of the more possible mechanisms for shaping nebulae is magnetic fields (e.g., García-Segura et al. 1999). 


\section{Summary}

We have used the VLA and ATCA to search for signatures of ionized winds - optically thick radio cores - in sixteen narrow-waist bipolar nebulae. Eleven nebulae were observed with the VLA at $1.3 \mathrm{~cm}$ and $0.7 \mathrm{~cm}$ with resolutions of $0.08^{\prime \prime}$ and $0.04^{\prime \prime}$, respectively. This is the first time that the centers of these objects have been systematically studied at such high angular resolution at radio wavelengths. Two objects, $19 \mathrm{~W} 32$ and M 1-91, were found to exhibit a compact central source with a positive spectral index. Three more objects examined with the VLA, NGC 6302, Hubble 5, and NGC 6537, exhibit extended radio emission that cannot be mapped properly at such short wavelengths. No emission was detected for six other objects in the VLA survey.

Five nebulae were observed with the ATCA at $6 \mathrm{~cm}$ and $3.6 \mathrm{~cm}$ with resolutions of $3^{\prime \prime}$ and $1.5^{\prime \prime}$, respectively. He 2-25 was found to have a compact central source only at $3.6 \mathrm{~cm}$. He 2-84 and $\mathrm{Mz} 3$ were found to contain a compact central source at both wavelengths. When short baselines that are sensitive to extended nebular emission were excluded, we found that the spectral index of these two radio cores changes with uv-coverage, suggesting that an optically thick core has been detected. In addition to its compact radio core, Mz 3 also exhibits extended nebulae emission that coincides with the two lobes seen in the HST optical images. He 2-36 also shows extended nebular emission but no compact core. No radio emission was detected for Th $2-\mathrm{B}$ in the ATCA study.

For 19W32 and M 1-91, we found that the predicted source sizes are smaller than the measured dimensions, which we attribute to the radio cores being elongated, implying a collimated wind or jet. The possibility that these radio cores represent COFs responsible for shaping the bipolar morphology of the nebulae is particularly intriguing. The derived mass loss rates of the wind from the radio observations are high in comparison to typical fast winds from central stars of planetary nebulae, and one possible solution is that the winds are not spherical symmetric but instead are collimated. If these winds are collimated by a binary system, then the radio imaging offers the best way to test such models. The five objects with radio core detections all exhibit a compact stellar-like optical core. This needs further investigation in order to understand the connection between the optical morphology and the radio ionized jets.

We are planning follow-up observations of these radio cores with higher angular resolution in order to study the structures of the cores. We have recently obtained observations of M 1-91 with the VLA A+PT (Pie Town) configuration. The addition of the Pie Town antenna will increase the resolution of the VLA by a factor of two while keeping the full sensitivity of the VLA. The new data will add to our knowledge of the core properties of M 1-91 once the analysis is completed. 
We thank the anonymous referee for constructive comments on the manuscript. We are grateful to the local staff of the NRAO, Narrabri and Siding Spring Observatories for their support during observations at the VLA, ATCA and SSO. The National Radio Astronomy Observatory is a facility of the National Science Foundation operated under cooperative agreement by Associated Universities, Inc. The Compact Array is part of the Australia Telescope, which is funded by the Commonwealth of Australia for operation as a National Facility managed by the Commonwealth Scientific and Industrial Research Organisation. The Siding Spring Observatory is operated by the Australian National University, Research School of Astronomy and Astrophysics.

THL expresses gratitude to the University of Calgary and the Province of Alberta for various scholarships and a travel grant during her $\mathrm{PhD}$. This work was supported in part by a grant to SK from the Natural Sciences and Engineering Research Council of Canada.

\section{REFERENCES}

Aaquist, O. B. \& Kwok, S. 1990, A\&AS, 84, 229

Allen, D. A., \& Swings, J. P. 1972, ApJ, 174, 583

Balick, B. 1987, AJ, 94, 671

Balick, B., \& Frank, A. 2002, ARA\&A, 40, 439

Bains, I., Redman, M. P., Bryce, M., \& Meaburn, J. 2004, MNRAS, 354, 549

Bujarrabal, V., Castro-Carrizo, A., Alcolea, J., \& Sánchez Contreras, C. 2001, A\&A, 377, 868

Corradi, R. L. M. 2003, in ASP Conf. Ser. 303, Symbiotic Stars Probing Stellar Evolution, eds. R. L. M. Corradi, J. Mikolajewska, \& T. J. Mahoney (San Francisco: ASP), 393

Corradi, R. L. M., \& Schwarz, H. E. 1995, A\&A, 293, 871

Doyle, S., Balick, B., Corradi, R. L. M., \& Schwarz, H. E. 2000, AJ, 119, 1339

Forveille, T., Huggins, P. J., Bachiller, R., \& Cox, P. 1998, ApJ, 495, L111

García-Segura, G., Langer, N., Różyczka, M., \& Franco, J. 1999, ApJ, 517, 767

Gieseking, F., Becker, I., \& Solf, J. 1985, ApJ, 295, L17

Goodrich, R. W. 1991, ApJ, 366, 163 
Huggins, P. J., Forveille, T., Bachiller, R., \& Cox, P. 2000, ApJ, 544, 889

Kohoutek, L. 1982, A\&A, 115, 420

Kohoutek, L., \& Surdej, J. 1980, A\&A, 85, 161

Kahn, F., \& West, K. A. 1985, MNRAS, 212, 837

Kwok, S. 2003a, in IAU Symp. 209: Planetary Nebulae: Their Evolution and Role in the Universe, eds. S. Kwok, M. Dopita, \& R. Sutherland (San Francisco: ASP), 3

Kwok, S. 2003b, in ASP Conf. Ser. 303, Symbiotic Stars Probing Stellar Evolution, eds. R. L. M. Corradi, J. Mikolajewska, \& T. J. Mahoney (San Francisco: ASP), 428

Kwok, S., Purton, C. R., \& Fitzgerald, P. M. 1978, ApJ, 219, L125

Kwok, S., Purton, C. R., Matthews, H. E., \& Spoelstra, T. A. T. 1985, A\&A, 144 ,321

Lee, T.-H., Stanghellini, L., Ferrario, L., \& Wickramasinghe, D. 2007, AJ, 133, in press (March)

Lim, J., \& Kwok, S. 2000, in ASP Conf. Ser. 199, Asymmetric Planetary Nebulae II: from Origins to Microstructures, eds. J. Kastner, S. Rappaport, \& N. Soker (San Francisco: ASP), 259

Lim, J., \& Kwok, S. 2003, in ASP Conf. Ser. 303, Symbiotic Starts Probing Stellar Evolution, eds. R. L. M. Corradi, J. Mikolajewska, \& T. J. Mahoney (San Francisco: ASP), 437

Livio, M. \& Soker, N. 2001, ApJ, 552, 685

López, J. A. 2002, Rev. Mex. Astron. Astrofis. Conf. Ser. 13, 139

López, J. A. 2003, in IAU Symp. 209, Planetary Nebulae and their Role in the Universe, eds. S. Kwok, M. Dopita, \& R. Sutherland (San Francisco: ASP), 483

Maciel, W. J. 1984, A\&AS, 55, 253

Manchado, A., Guerrero, M. A., Stanghellini, L., \& Serra-Ricart, M. 1996, The IAC morphological catalog of northern galactic planetary nebulae (La Laguna: Instituto de Astrofísica de Canarias)

Massey, P. 1997, A User's Guide to CCD Reductions with IRAF

Merrill, P. W. 1919, PASP, 31, 305 
Merrill, P. W. 1958, in Etoiles à Raies d'Emission (cointe-Sclessin: Institut d'Astrophysique), 20, 436

Merrill, P. W., \& Hummason, M. L. 1932, PASP, 44, 56

National Radio Astronomy Observatory 2003. The AIPS Cookbook (Charlottesville: NRAO)

Osterbrock, D. E. 1989, Astrophysics of Gaseous Nebulae and Active Galactic Nuclei (Mill Valley: Univ. Science Books)

Panagia, N., \& Felli, M. 1975, A\&A, 39, 1

Pauldrach, A.W.A., Hoffmann, T.L., \& Méndez, R.H. 2003, in IAU Symp. 209: Planetary Nebulae: Their Evolution and Role in the Universe, eds. S. Kwok, M. Dopita, \& R. Sutherland (San Francisco: ASP), 177

Perinotto, M. 1983, in IAU Symp. 103, Planetary Nebulae, ed. D. R. Flower (Dordrecht: D. Reidel Publishing Co.), 323

Phillips, J. P. 2004, MNRAS, 353, 589

Plaskett, J. S. 1928, Publ. Dom. Astrophys. Obs. Victoria, 4, 119

Reid, M. J., \& Menten, K. M. 1997, ApJ, 476, 327

Reynolds, S. P. 1986, ApJ, 304, 713

Sault, R. J., \& Killeen, N. 2003, Miriad Users Guide, Australia Telescope National Facility

Soker, N., \& Rappaport, S. 2000, ApJ, 538, 241

van der Veen, W. E. C. J., Habing, H. J., \& Geballe, T. R. 1989, A\&A, 226, 108

Wright, A. E., \& Barlow, M. J. 1975, MNRAS, 170, 41

Zijlstra, A. A., Pottasch, S. R., \& Bignell, C. 1989, A\&AS, 79, 329

This preprint was prepared with the AAS IATEX macros v5.2. 
Table 1. The properties of the selected PNs with the first 11 objects observed by the VLA and the last 5 objects observed by the ATCA.

\begin{tabular}{ccccccc}
\hline \hline Object & PN G & RA & Dec & VNW & Optical core & Point/Mirror \\
\hline IRAS 07131-0147 & $\ldots$ & $07: 15: 42.6$ & $-01: 52: 42$ & yes & yes & $?$ \\
M 1-16 & $226.7+05.6$ & $07: 37: 18.6$ & $-09: 38: 48$ & $?$ & $?$ & yes \\
NGC 2818 & $261.9+08.5$ & $09: 16: 01.5$ & $-36: 37: 37$ & $?$ & no & $?$ \\
NGC 6302 & $349.5+01.0$ & $17: 13: 44.2$ & $-37: 06: 14$ & yes & no & no \\
19W32 & $359.2+01.2$ & $17: 39: 02.8$ & $-28: 56: 35$ & yes & yes & $?$ \\
HB 5 & $359.3-00.9$ & $17: 47: 56.8$ & $-29: 59: 53$ & yes & yes & yes \\
NGC 6537 & $010.1+00.7$ & $18: 05: 13.2$ & $-19: 50: 34$ & yes & $?$ & yes \\
M 3-28 & $021.8-00.4$ & $18: 32: 41.3$ & $-10: 06: 05$ & yes & $?$ & yes \\
M 1-91 & $061.3+03.6$ & $19: 32: 57.3$ & $+26: 52: 43$ & yes & yes & yes \\
M 2-48 & $062.4-00.2$ & $19: 50: 27.9$ & $+25: 54: 28$ & yes & $?$ & no \\
NGC 7026 & $089.0+00.3$ & $21: 06: 18.5$ & $+47: 51: 08$ & $?$ & $?$ & yes \\
He 2-25 & $275.2-03.7$ & $09: 18: 01.3$ & $-54: 39: 29$ & yes & yes & $?$ \\
He 2-36 & $279.6-03.1$ & $09: 43: 25.6$ & $-57: 16: 56$ & $?$ & yes & yes \\
He 2-84 & $300.4-00.9$ & $12: 28: 46.7$ & $-63: 44: 35$ & yes & yes & yes \\
Th 2-B & $\ldots$ & $13: 28: 38.2$ & $-63: 49: 42$ & $?$ & yes & $?$ \\
Mz 3 & $331.7-01.0$ & $16: 17: 12.6$ & $-51: 59: 08$ & yes & yes & yes
\end{tabular}

Table 2. Optical observing parameters

\begin{tabular}{cccc}
\hline \hline Filter Name & $\begin{array}{c}\text { Center } \lambda \\
(\AA)\end{array}$ & $\begin{array}{c}\text { Bandwidth } \Delta \lambda \\
(\AA)\end{array}$ & $\begin{array}{c}\text { FWHM } \\
\left({ }^{\prime \prime}\right)\end{array}$ \\
\hline $\mathrm{H} \alpha$ & 6568 & 8 & 2.2 \\
{$[\mathrm{~N} \mathrm{II}]$} & 6588 & 8.8 & 2.4 \\
{$[\mathrm{O} \mathrm{III}]$} & 5007 & 10 & 2.4 \\
\hline
\end{tabular}


Table 3. Results of the VLA and ATCA Surveys.

\begin{tabular}{cccccc}
\hline \hline Object & $\begin{array}{c}\text { Detection limit } \\
(J y)\end{array}$ & \multicolumn{2}{c}{ Synthesized beam size } & Type of Detection \\
& & \multicolumn{2}{c}{$\left({ }^{\prime \prime}\right)$} & \\
\hline IRAS 07131-0147 & $3.0 \mathrm{E}-4$ & $7.2 \mathrm{E}-4$ & $0.11 \times 0.079$ & $0.080 \times 0.041$ & \\
M 1-16 & $3.3 \mathrm{E}-4$ & $7.8 \mathrm{E}-4$ & $0.13 \times 0.077$ & $0.080 \times 0.042$ & $\ldots$ \\
NGC 2818 & $5.1 \mathrm{E}-4$ & $1.5 \mathrm{E}-3$ & $0.26 \times 0.082$ & $0.16 \times 0.042$ & $\ldots$ \\
NGC 6302 & $6.6 \mathrm{E}-4$ & $4.2 \mathrm{E}-3$ & $0.26 \times 0.083$ & $0.17 \times 0.041$ & extended \\
19W32 & $3.6 \mathrm{E}-4$ & $9.9 \mathrm{E}-4$ & $0.19 \times 0.082$ & $0.12 \times 0.040$ & compact \\
HB 5 & $3.9 \mathrm{E}-4$ & $9.6 \mathrm{E}-4$ & $0.20 \times 0.078$ & $0.12 \times 0.042$ & extended \\
NGC 6537 & $3.9 \mathrm{E}-4$ & $9.9 \mathrm{E}-4$ & $0.16 \times 0.077$ & $0.094 \times 0.043$ & extended \\
M 3-28 & $2.9 \mathrm{E}-4$ & $7.2 \mathrm{E}-4$ & $0.14 \times 0.079$ & $0.080 \times 0.046$ & $\ldots$ \\
M 1-91 & $3.0 \mathrm{E}-4$ & $8.1 \mathrm{E}-4$ & $0.10 \times 0.083$ & $0.061 \times 0.045$ & compact \\
M 2-48 & $3.0 \mathrm{E}-4$ & $7.5 \mathrm{E}-4$ & $0.10 \times 0.084$ & $0.060 \times 0.045$ & $\ldots$ \\
NGC 7026 & $3.3 \mathrm{E}-4$ & $8.1 \mathrm{E}-4$ & $0.096 \times 0.091$ & $0.068 \times 0.042$ & $\ldots$ \\
\hline He 2-25 & $6 \mathrm{~cm}$ & $3.6 \mathrm{~cm}$ & $6 \mathrm{~cm}$ & $3.6 \mathrm{~cm}$ & \\
He 2-36 & $3.3 \mathrm{E}-4$ & $1.9 \mathrm{E}-4$ & $3.74 \times 2.71$ & $1.98 \times 1.42$ & compact \\
He 2-84 & $1.8 \mathrm{E}-4$ & $1.8 \mathrm{E}-4$ & $3.59 \times 2.87$ & $1.88 \times 1.52$ & extended \\
Th 2-B & $1.7 \mathrm{E}-4$ & $2.1 \mathrm{E}-4$ & $3.45 \times 3.02$ & $1.76 \times 1.60$ & compact \\
Mz 3 & $1.7 \mathrm{E}-4$ & $2.1 \mathrm{E}-4$ & $3.47 \times 2.71$ & $1.82 \times 1.42$ & $\ldots$ \\
\hline & $4.5 \mathrm{E}-4$ & $4.5 \mathrm{E}-4$ & $4.09 \times 2.11$ & $2.24 \times 1.16$ & compact + extended \\
\hline
\end{tabular}

Table 4. Flux densities for compact cores of 19W32 and M 1-91

\begin{tabular}{lcccl}
\hline \hline Object & Fitting Process & $\begin{array}{c}1.3 \mathrm{~cm} \\
(\mathrm{mJy})\end{array}$ & $\begin{array}{c}0.7 \mathrm{~cm} \\
(\mathrm{mJy})\end{array}$ & Spectral Index \\
\hline \multirow{2}{*}{$19 \mathrm{~W} 32$} & Visibility fit & $3.58 \pm 0.18$ & $6.18 \pm 0.69$ & $0.81 \pm 0.18$ \\
& Map fit & $3.48 \pm 0.17$ & $4.76 \pm 0.57$ & $0.47 \pm 0.19$ \\
\hline \multirow{2}{*}{ M 1-91 } & Visibility fit & $2.97 \pm 0.16$ & $5.11 \pm 0.39$ & $0.79 \pm 0.14$ \\
& Map fit & $2.65 \pm 0.15$ & $3.97 \pm 0.38$ & $0.64 \pm 0.17$ \\
\hline
\end{tabular}


Table 5. Flux densities for compact cores of He 2-84 and Mz 3

\begin{tabular}{|c|c|c|c|c|}
\hline Object & Fitting Process & $\begin{array}{c}6 \mathrm{~cm} \\
(\mathrm{mJy})\end{array}$ & $\begin{array}{c}3.6 \mathrm{~cm} \\
(\mathrm{mJy})\end{array}$ & Spectral Index \\
\hline He $2-25$ & Map pixel sum & $\leq 0.33$ & $0.69 \pm 0.06$ & $\gtrsim+1.25$ \\
\hline \multirow{2}{*}{ He 2-84 } & Visibility fit & $12.1 \pm 0.16$ & $9.2 \pm 0.16$ & $-0.47 \pm 0.04$ \\
\hline & Map fit & $8.6 \pm 0.06$ & $7.7 \pm 0.08$ & $-0.18 \pm 0.02$ \\
\hline $\mathrm{Mz} 3$ & Map pixel sum & $15.3 \pm 0.15$ & $18.3 \pm 0.15$ & $+0.35 \pm 0.02$ \\
\hline
\end{tabular}

Table 6. Compact core fluxes vs. uv-range for He 2-84 and Mz 3

\begin{tabular}{ccccc}
\hline \hline Object & $\begin{array}{c}u v \text {-range } \\
(\mathrm{k} \lambda)\end{array}$ & $\begin{array}{c}6 \mathrm{~cm} \\
(\mathrm{mJy})\end{array}$ & $\begin{array}{c}3.6 \mathrm{~cm} \\
(\mathrm{mJy})\end{array}$ & Spectral Index \\
\hline & $50-100$ & $1.83 \pm 0.14$ & $2.60 \pm 0.10$ & $+0.60 \pm 0.15$ \\
$\mathrm{He} 2-84$ & $65-100$ & $1.39 \pm 0.16$ & $1.89 \pm 0.12$ & $+0.52 \pm 0.22$ \\
& $75-100$ & $1.08 \pm 0.18$ & $1.42 \pm 0.15$ & $+0.47 \pm 0.34$ \\
\hline \multirow{2}{*}{$\mathrm{Mz} 3$} & $45-100$ & $13.3 \pm 0.11$ & $19.5 \pm 0.11$ & $+0.65 \pm 0.02$ \\
& $55-100$ & $10.8 \pm 0.14$ & $20.3 \pm 0.11$ & $+1.07 \pm 0.02$ \\
\hline
\end{tabular}


Table 7. Mass loss rates and distances of the five objects with radio core detection

\begin{tabular}{cccc}
\hline \hline Object & $\begin{array}{c}\dot{M}_{v D^{\dagger}} \\
\left(M_{\odot \mathrm{yr}^{-1}}\right)\end{array}$ & $\begin{array}{c}\text { Distance } \\
(\mathrm{kpc})\end{array}$ & D. Reference \\
\hline 19W32 & $\sim 5 \times 10^{-9}$ & 3.0 & 1 \\
M 1-91 & $\sim 5 \times 10^{-9}$ & 7.0 & 2 \\
He 2-25 & $\sim 2 \times 10^{-9}$ & 2.1 & 3 \\
He 2-84 & $\sim 5 \times 10^{-9}$ & 1.6 & 2 \\
Mz 3 & $\sim 3 \times 10^{-8}$ & 3.3 & 4 \\
\hline
\end{tabular}

$$
{ }^{\dagger} \dot{M}\left(M_{\odot} \mathrm{yr}^{-1}\right)=\dot{M}_{v D} \times\left(v / \mathrm{km} \mathrm{s}^{-1}\right)(D / \mathrm{kpc})^{3 / 2}
$$

References. - 1. Kohoutek (1982); 2. Maciel (1984); 3. Phillips (2004); 4. van der Veen et al. (1989).

Table 8. The measured dimensions and predicted sizes of 19 W32 and M 1-91

\begin{tabular}{lccccc}
\hline \hline Object & $\begin{array}{c}\lambda \\
(\mathrm{cm})\end{array}$ & $\begin{array}{c}\text { Resolved Axis } \\
(\mathrm{mas})\end{array}$ & $\begin{array}{c}\text { P.A. }^{\mathrm{a}} \\
\left({ }^{\circ}\right)\end{array}$ & $\begin{array}{c}r(\nu) \\
(\mathrm{mas})\end{array}$ & $\begin{array}{c}\theta_{0} \\
\left.{ }^{\circ}\right)\end{array}$ \\
\hline \multirow{2}{*}{ 19W32 } & 1.3 & $63 \pm 11$ & $51 \pm 19$ & 17 & 7.8 \\
& 0.7 & $51 \pm 10$ & $56 \pm 46$ & 10 & 4.3 \\
\hline \multirow{2}{*}{ M 1-91 } & 1.3 & $77 \pm 7$ & $86 \pm 6$ & 14 & 4.0 \\
& 0.7 & $34 \pm 6$ & $58 \pm 12$ & 9 & 8.0 \\
\hline
\end{tabular}

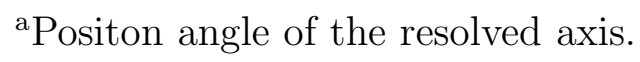



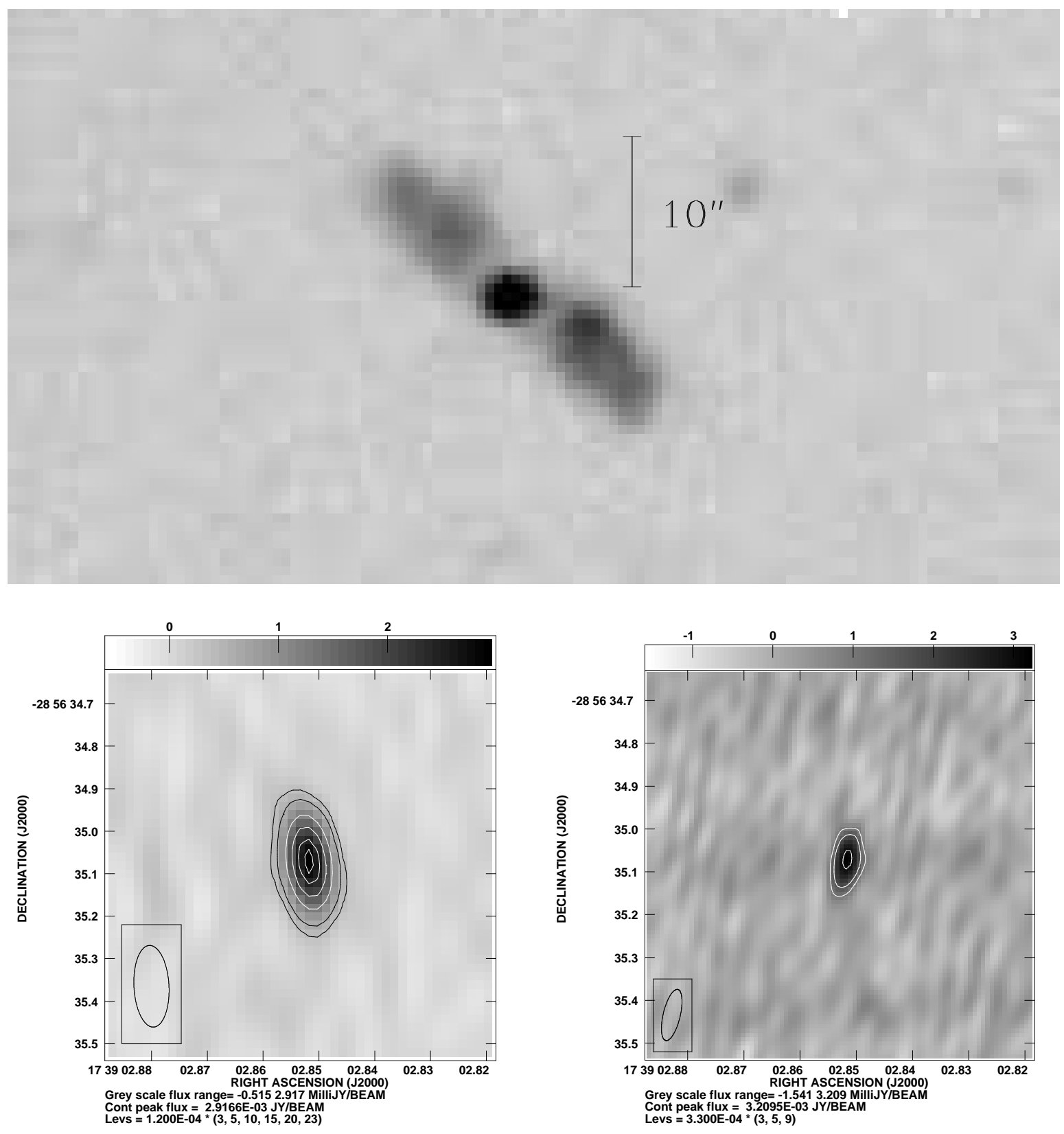

Fig. 1.- Optical and radio images of 19W32. a) SSO $\mathrm{H} \alpha$ image in a linear scale. b) VLA $1.3 \mathrm{~cm}$ (left) and $0.7 \mathrm{~cm}$ (right) radio continuum images with contours superposed. The contours start at $3 \sigma$, with the noise level $\sigma=1.1 \times 10^{-4} \mathrm{Jy} / \mathrm{beam}$ at $1.3 \mathrm{~cm}$ and $\sigma=3.6 \times 10^{-4} \mathrm{Jy} /$ beam at $0.7 \mathrm{~cm}$. The radio images are on a much smaller scale, with the axis range only $\sim 1^{\prime \prime}$. The position of the radio core coincides with the optical stellar core. In this and subsequent figures, the ellipse in the box in the lower-left corner represents the half-power extent of the synthesized beam. 

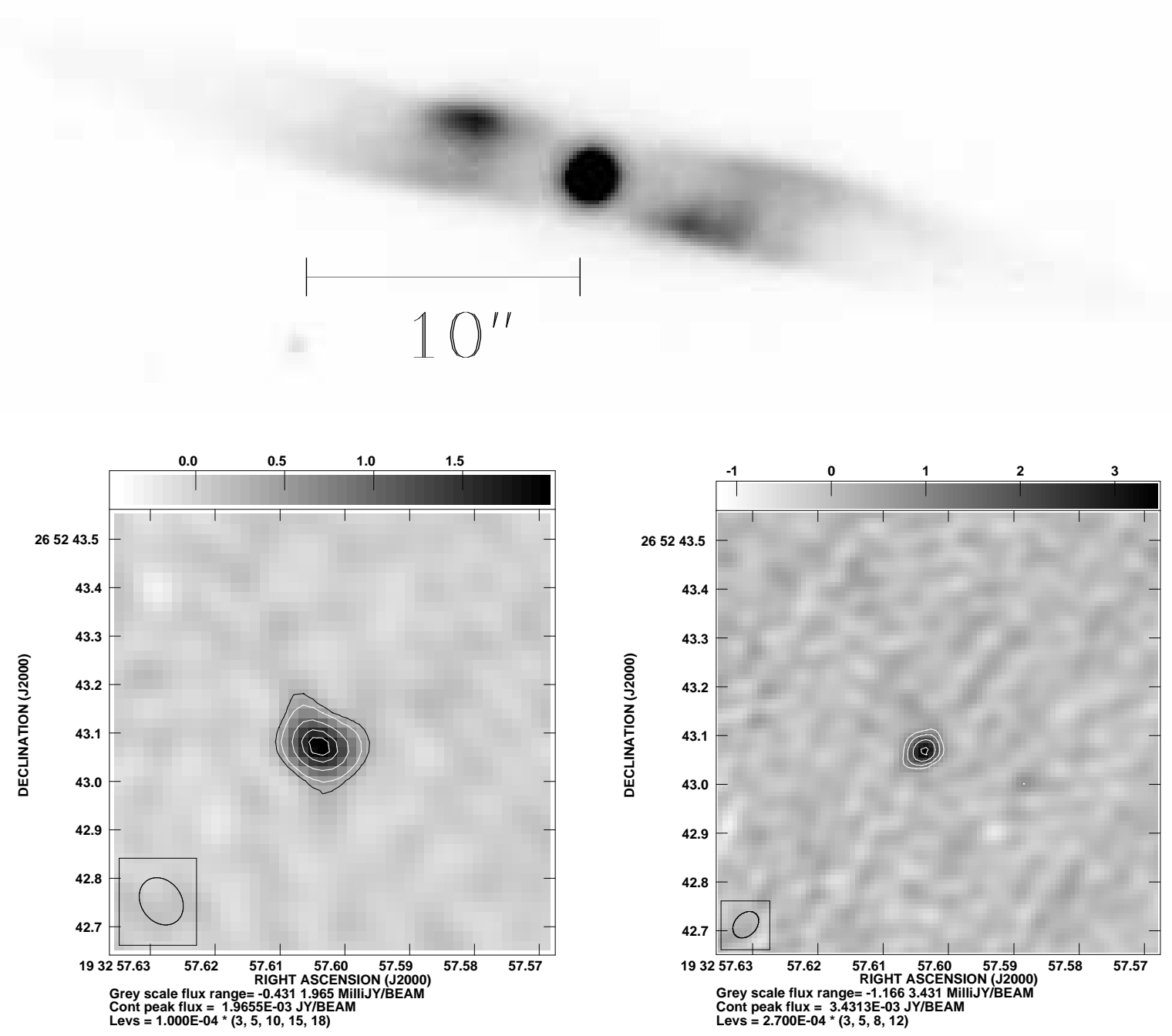

Fig. 2.- Optical and radio images of $\mathrm{M}$ 1-91. a) IAC $\mathrm{H} \alpha$ image in a linear scale. b) VLA $1.3 \mathrm{~cm}$ (left) and $0.7 \mathrm{~cm}$ (right) radio continuum image with contours superposed. The contours start at $3 \sigma$, with the noise level $\sigma=9.95 \times 10^{-5} \mathrm{Jy} / \mathrm{beam}$ at $1.3 \mathrm{~cm}$ and $\sigma=2.7 \times 10^{-4} \mathrm{Jy} /$ beam at $0.7 \mathrm{~cm}$. The radio images are on a much smaller scale, with the axis range only $\sim 1^{\prime \prime}$. The position of the radio core coincides with the optical stellar core. 

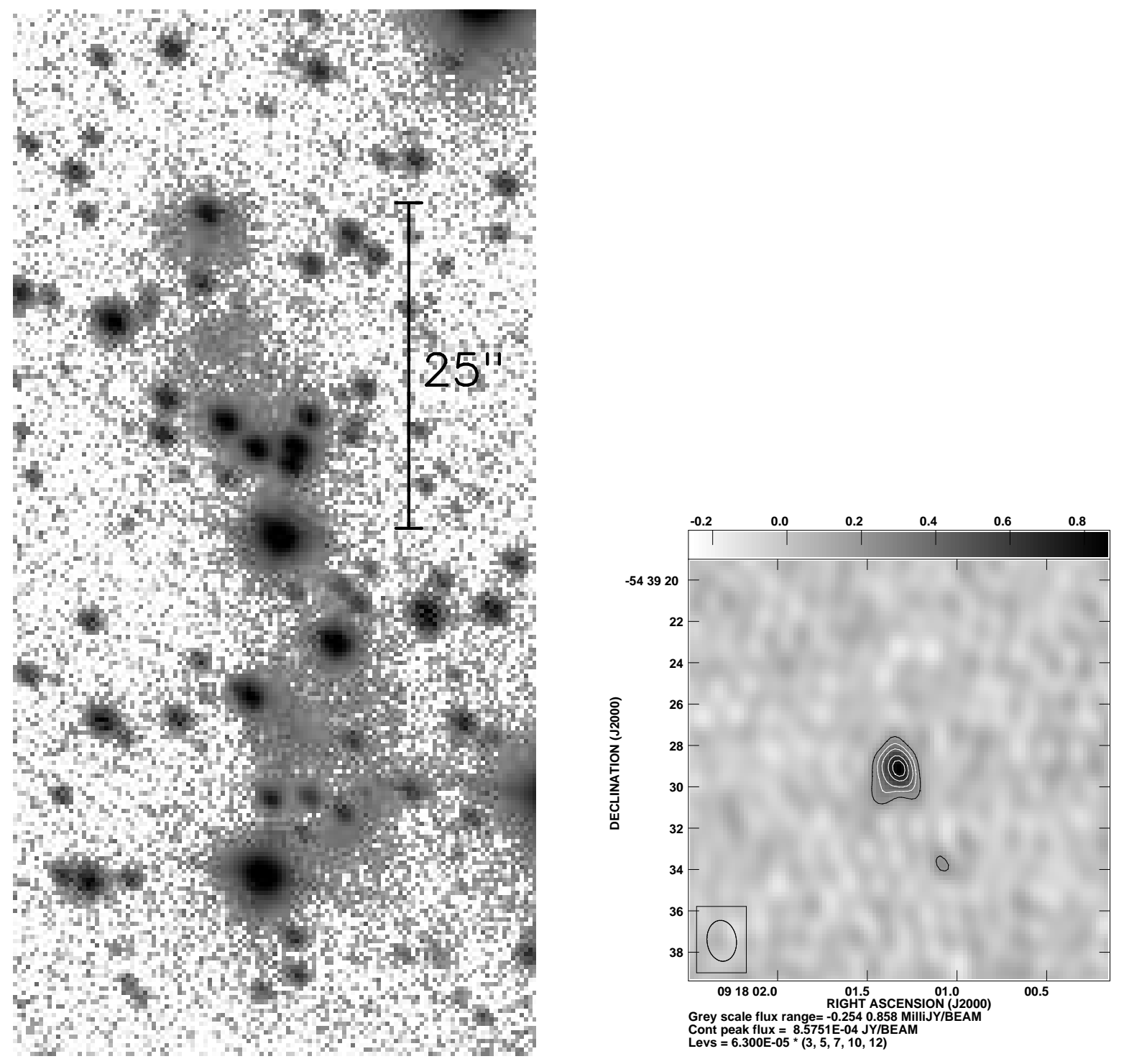

Fig. 3.- Optical and radio images of He 2-25. a) NTT R-band image (Lee et al. 2006) in a logarithmic intensity scale. b) ATCA $3.6 \mathrm{~cm}$ (right) radio continuum image in greyscale with contours superposed. The contours start at $3 \sigma$ with the noise level $\sigma=6.3 \times 10^{-5}$ $\mathrm{Jy} /$ beam at $3.6 \mathrm{~cm}$. No emission is detected at $6 \mathrm{~cm}$. 

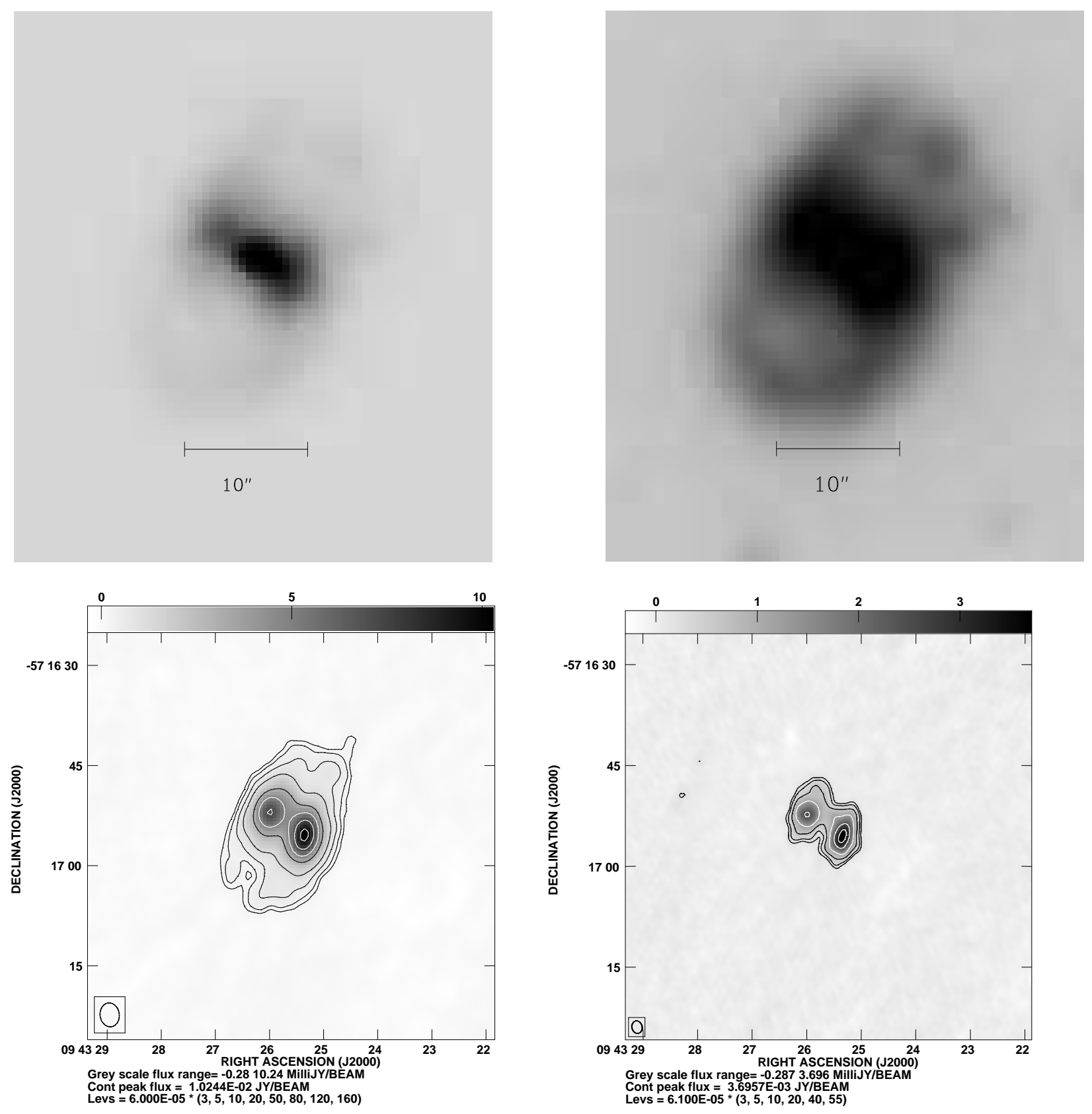

Fig. 4.- Optical and radio images of $\mathrm{He}$ 2-36. a) $\mathrm{SSO} \mathrm{H} \alpha$ images show the detailed structures of the center in a linear scale (left) and the outer lobes in a logarithmic intensity scale (right). b) ATCA $6 \mathrm{~cm}$ (left) and $3.6 \mathrm{~cm}$ (right) radio continuum image in greyscale with contours superposed. The contours start at $3 \sigma$ with the noise level $\sigma=6.0 \times 10^{-5}$ $\mathrm{Jy} /$ beam at $6 \mathrm{~cm}$ and $\sigma=6.1 \times 10^{-5} \mathrm{Jy} /$ beam at $3.6 \mathrm{~cm}$. 

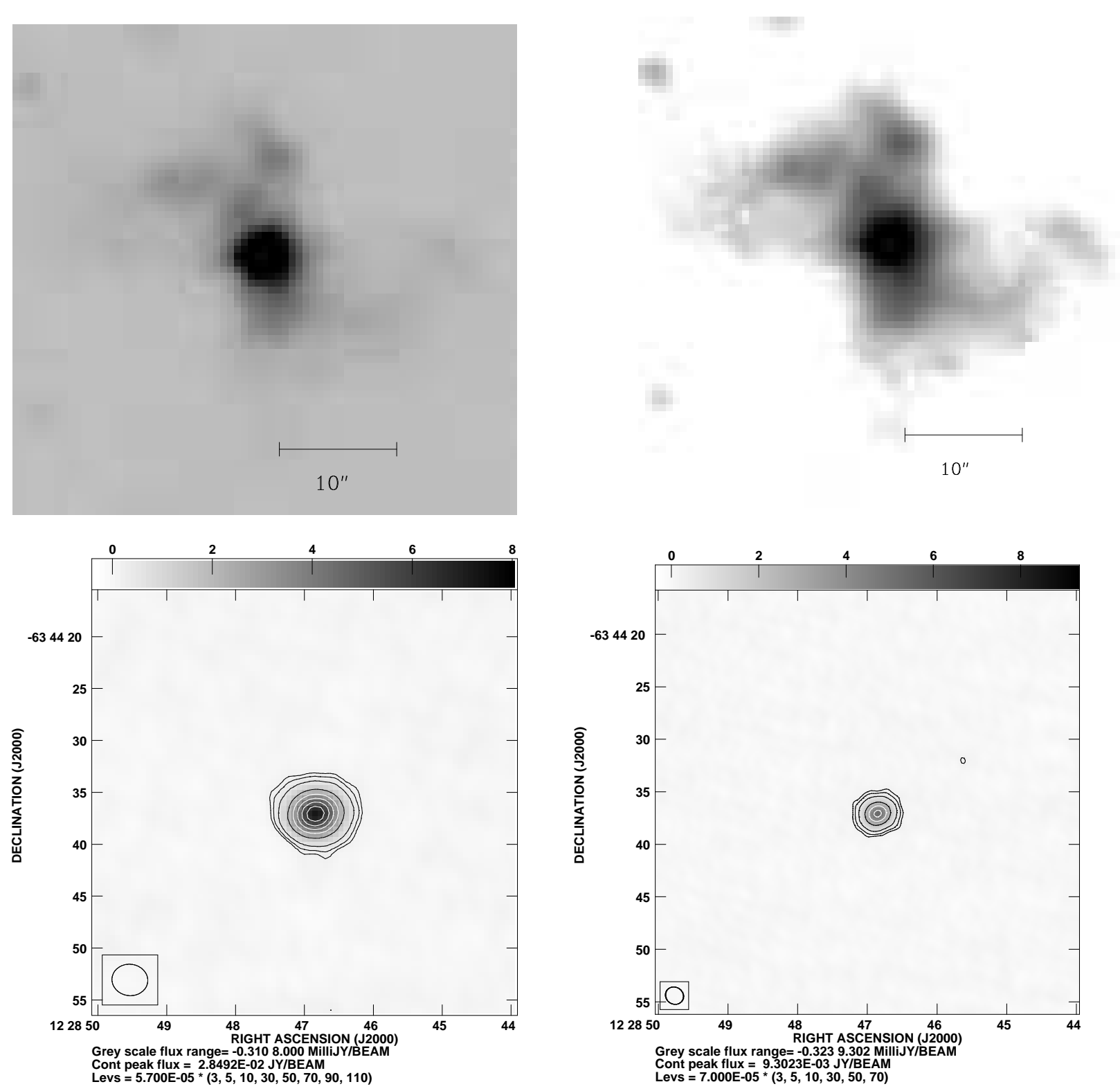

Fig. 5.- Optical and radio images of $\mathrm{He}$ 2-84. a) $\mathrm{SSO} \mathrm{H} \alpha$ images show the detailed structures of the center in a linear scale (left) and the outer lobes in a logarithmic intensity scale (right). b) ATCA $6 \mathrm{~cm}$ (left) and $3.6 \mathrm{~cm}$ (right) radio continuum image in greyscale with contours superposed. The contours start at $3 \sigma$ with the noise level $\sigma=5.7 \times 10^{-5}$ $\mathrm{Jy} /$ beam at $6 \mathrm{~cm}$ and $\sigma=7.0 \times 10^{-5} \mathrm{Jy} /$ beam at $3.6 \mathrm{~cm}$. The position of the radio source coincides with the optical stellar core. 

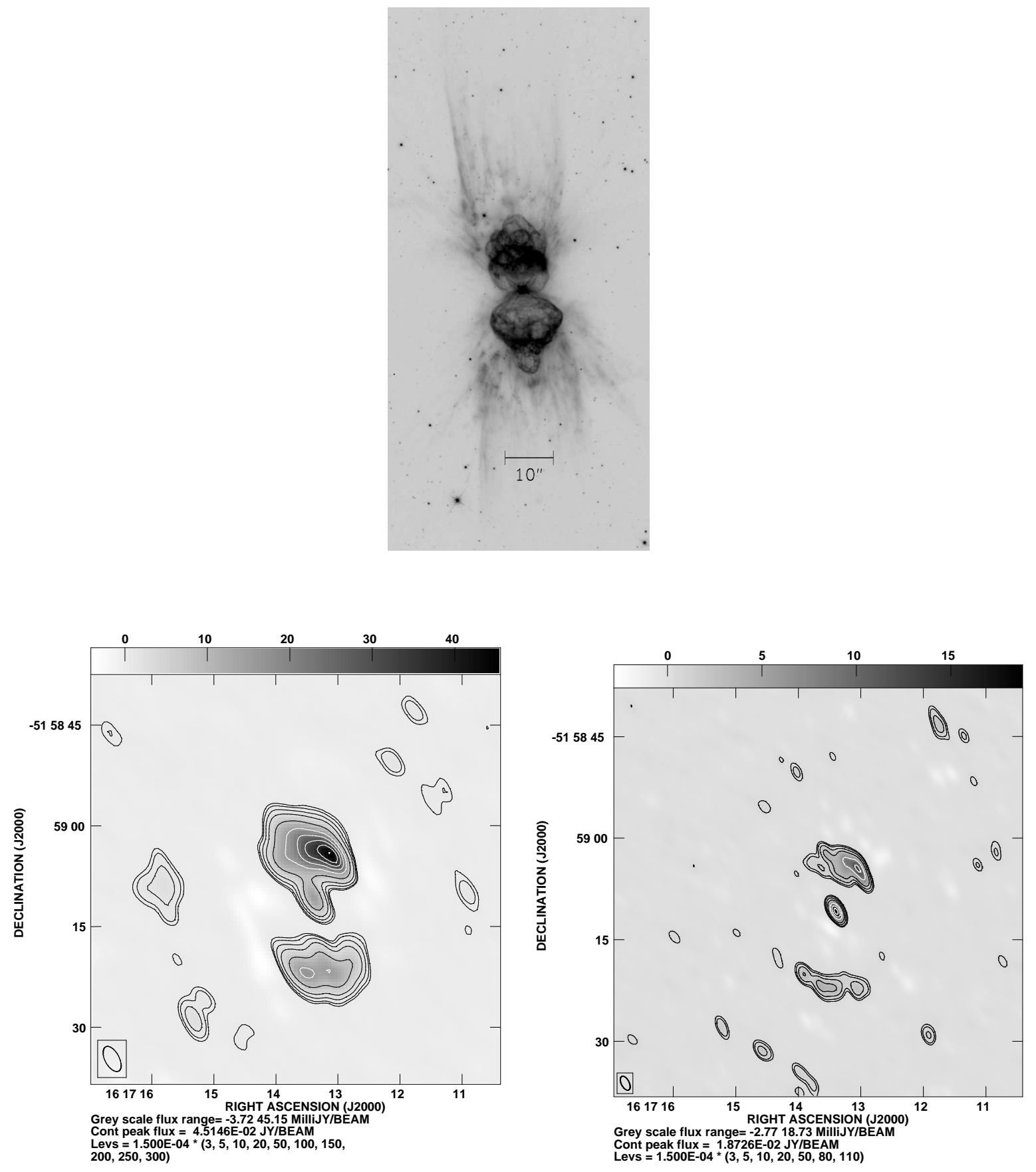

Fig. 6.- Optical and radio images of $\mathrm{Mz}$ 3. a) $H S T \mathrm{H} \alpha$ image in logarithmic scales. b) ATCA $6 \mathrm{~cm}$ (left) and $3.6 \mathrm{~cm}$ (right) radio continuum image in greyscale with contours superposed. The contours start at $3 \sigma$ with the noise level $\sigma=1.5 \times 10^{-4} \mathrm{Jy} / \mathrm{beam}$ at $6 \mathrm{~cm}$ and $\sigma=1.5 \times 10^{-4} \mathrm{Jy} /$ beam at $3.6 \mathrm{~cm}$. The radio core and bipolar lobes coincide with the optical stellar core and the brightest structures of the optical lobes. 

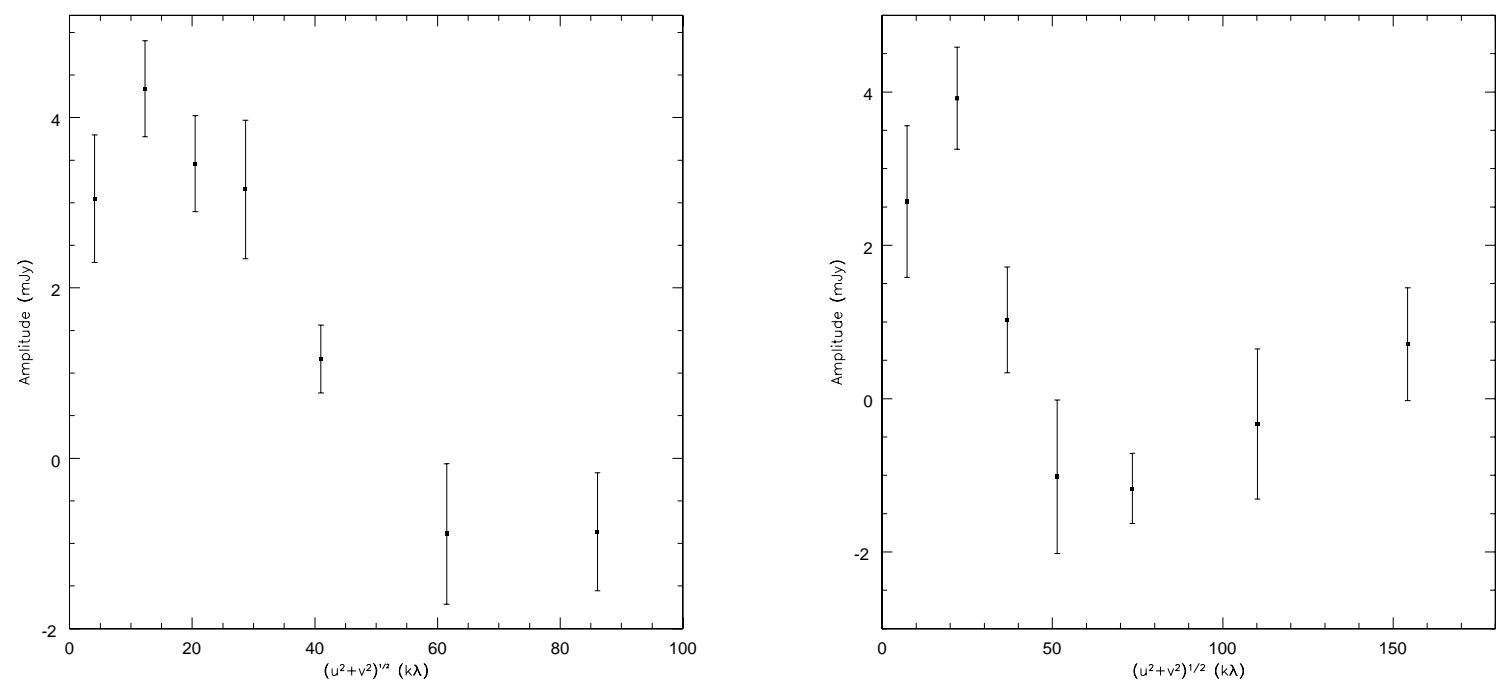

Fig. 7.- Amplitude vs. uv-distance for He 2-84 at $6 \mathrm{~cm}$ (left) and $3.6 \mathrm{~cm}$ (right). The real component of the ampplitudes are averaged in several $u v$ bins. The binning size is wider in the longer $u v$-distance in order to increase the signal to noise ratio at longer baselines.
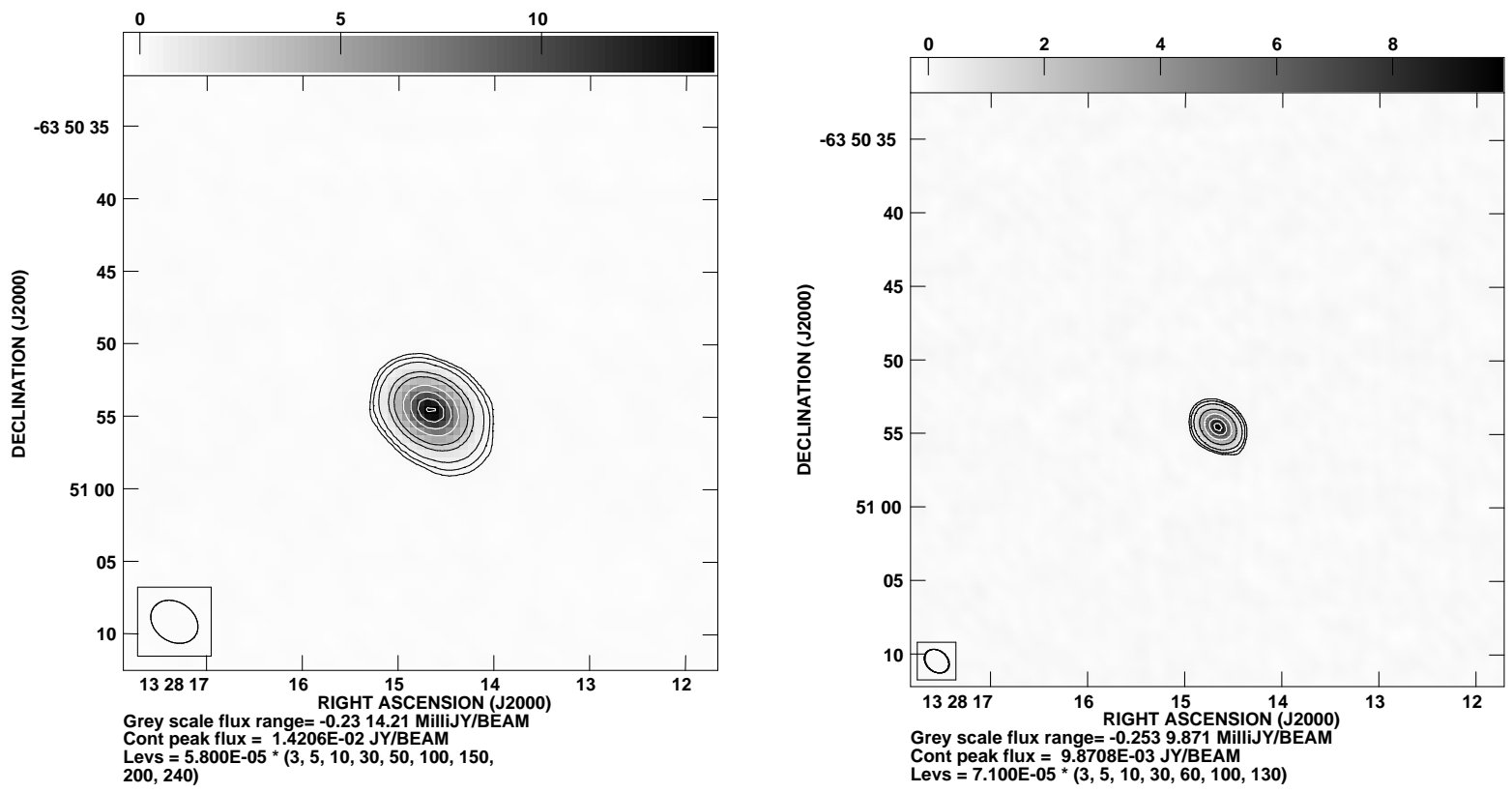

Fig. 8. - Images of a background radio source near Th 2-B at $6 \mathrm{~cm}$ (left) and $3.6 \mathrm{~cm}$ (right). 